\title{
Posiciones Romanísticas en Torno a la Solidaridad Natural y Jurídica de la Prestación de Alimentos entre Hermanos
}

\author{
Juan Miguel Alburquerque \\ Professor catedrático de Direito Romano na \\ Faculdade de Direito da Universidade de Córdoba \\ (Espanha) \\ fdlalsaj@uco.es
}

Sumario: Introduccion. fuentes. exégesis particular y doctrinal. 1. Deber explícito: prestar los alimentos necesarios a la hermana (o hermano). 2. La relación de alimentos podría considerarse como una auténtica relación jurídica. 3. Reflexiones y observaciones.

Resumen: En este apartado destacamos el carácter de obligación que tiene la prestación de alimentos entre hermanos, probablemente ya desde la época clásica más avanzada. Apoyan nuestra idea un conjunto lineal de estimaciones jurisprudenciales afirmativas y el análisis profundo de las fuentes jurídicas principales.

Palabras clave: Alimenta. Victus. Filius.

\section{INTRODUCCIÓN. FUENTES. EXÉGESIS PARTICULAR Y DOCTRINAL}

Presuponer la existencia de una obligación recíproca de alimentos entre hermanos, ya incluso en la etapa clásica imperial, puede ser, en nuestra opinión, al menos atendible ${ }^{1}$. Hablamos de una estimación no resuelta convincentemente por otros estudiosos que han confiado decididamente en otras tentativas exegéticas, pero que tampoco han supuesto la anulación de las dudas existentes.

\footnotetext{
${ }^{1}$ Cfr., ALBURQUERQUE, J.M., Patria potestas in pietate debet, non atrocitate consistere. Iuris Tantum n ${ }^{\circ}$ 16. Universidad Anáhuac. México junio 2005; Id. De la justicia y la reciprocidad en situaciones de necesidad y dependencia: El reconocimiento y la inclusión jurídico-social de la madre en el cumplimiento de la obligación
} 
A favor de nuestra idea podríamos interpretar los siguientes textos con las opiniones de Juliano, Ulpiano, Gayo y Paulo.

En efecto, en D. 27,2, 4, Juliano parece que admite la obligación del hermano de proporcionar alimentos a su hermana. Entre las motivaciones insuficientes que se dan para justificar la interpolación del mismo, podríamos recordar ahora la de BESELER ${ }^{2}$ -que ha sido subrayada por $\mathrm{BIONDI}^{3}$ para destacar su simplicidad y, quizá, su escasa solidez-, "Wohl christlige Neurung Justinians":

de alimentos respecto a los hijos, RGDR 11 www.iustel.com, Madrid 2009; Id. Prestación de alimentos entre parientes en Derecho romano. Atención a las necesidades más primarias y su aparente evolución. Iuris Tantum $n^{\circ} 17$ Universidad Anáhuac. México diciembre 2006; Id. Realidad social o jurídica de la prestación de alimentos entre cónyuges, en RGDR 10 junio 2008, pp. 1 y ss.; Id. La prestación de alimentos entre pariente. Introducción y antecedentes como deber moral, en Personalidad y capacidad jurídicas, vol. I, Córdoba 2005, pp.89 y ss.; Id. Deber legal u obligación moral originaria: Generalidades introductorias sobre la prestación de alimentos en derecho romano, RGDR 3 diciembre 2004, pp. 1 y ss.; Id. Alimentos entre parientes (II): alimenta et victus. Puntualizaciones breves sobre la transacción y la prestación en el marco de los posibles procedimientos (expedientes) de jurisdicción voluntaria, RGDR 4 junio 2005; Id. Aproximación a la perspectiva jurisprudencial sobre el contenido de la prestación de alimentos derivada de una relación de parentesco, en Anuario da Facultade de Dereito da Universidade da Coruña 9, A Coruña 2005, pp. 13 y ss.; Id. Notas, conjeturas e indicios previos a la regulación de Antonino Pio y Marco Aurelio, RGDR 6 junio 2006; Id. Aspectos de la prestación de alimentos en derecho romano: Especial referencia a la reciprocidad entre padre e hijo, ascendientes y descendientes, en Revista Jurídica de la Universidad Autónoma de Madrid RJUAM nº 15, 2007. Cfr., sobre alimentos y parentesco, entre otros, Cfr. FERNÁNDEZ DE BUJÁN, A., El filiusfamilias independiente en Roma y en el derecho español, Madrid 1984, pp. 21 y ss.; Id. Derecho Público Romano. Recepción, Jurisdicción y Arbitraje, $12^{a}$ ed., 2009, pp. 99 y ss; Id. Reflexiones a propósito de la realidad social, la tradición jurídica y la moral cristiana en el matrimonio romano (I), en RGDR ${ }^{\circ} 6$, junio 2006; Id. Derecho Privado Romano, 2a ed. Iustel, 2009 pp. 117 y ss.; FERNÁNDEZ DE BUJÁN, FEDERICO., La vida, principio rector del derecho, 1999, pp. 101 y ss., y 151 y ss.; GARCÍA GARRIDO, M.J., Ius uxorium. El régimen patrimonial de la mujer casada en derecho romano, Roma-Madrid 1958, pp. 93 y ss; SACHERS, E., Das Recht auf Unterhalt in der römischen Familie der klass. Zeit, Festschrift Fritz Schulz, Weimar, 1951, vol. I, pp. 310 y ss.; Id. Potestas patria, RE., 22, pp. 1114 y ss.; ALBERTARIO, E., Studi di diritto romano, vol I, Persone e famiglia, Milano 1933, especialmente el capítulo XIII, Sul diritto agli alimenti, pp. 251. (Proviene del artículo incluido en Pubblicazioni dell'Universtà Cattolica del S. Cuore de 1925); LONGO, G., Sul diritto agli alimenti, Annali Univ. Macerata, 1948, vol. XVII, pp. 215 y ss.; (= Ricerche Romanistiche, Milano 1966 pp. 339 y ss); SOLAZZI, S., La prestazione degli alimenti. En Scritti di diritto romano III, (1925-1937), Napoli 1960, pp. 127 y ss.; LAVAGGI, G., Alimenti (diritto romano), cit.,pp. 18 y ss.; LENEL, O., Das Edictum Perpetuum. Ein Versuch zu seiner Wiederherstellung, $3^{a}$ edición, Leipzig 1927, p. 488 (reimpresión Aalen 1985).; Id. Palingenesia Iuris Civilis, reimp. de 1960, 2, 953 (Scientia Verlag Aalen 2000); BESELER, G., Beiträgt zur Kritik der römischen Rechtsquellen I-IV; ZOZ, M.G., In tema di obbligazioni alimentari, BIDR 73. Milano 1970, pp. 324 y ss. Id. Sulla capacità a ricevere fedecommessi alimentari, SDHI 40, 1974, Roma 1974, pp. 303 y ss.

2 BESELER, G., Beiträgt zur Kritik der römischen Rechtsquellen, cit., 2, p. 42. ALBERTARIO, E., Sul diritto agli alimenti, cit., pp. 270 y ss., niega la base sustancial clásica de una obligación recíproca entre hermanos; Otros, ni siquiera la admiten en derecho justinianeo (GLÜCK, Commentario alle pandette, 25 1290 a, 266 y ss.)

3 BIONDI, B., Alimenti, cit., p. 293. 
D. 27,2,4 (I ulianus, libro XXI digestorum): Q ui fiium heredem instituerat, filae dotis nomine, cum in familia nupsisset, ducenta legaverat nec quicquam praeterea, et tutorem eis Sempronium dedit: is a cognatis et a propinquis pupillae perductus ad magistratum iussus est al imenta pupillae et mercedes, ut liberalibus artibus institueretur, pupillae nomine praeceptoribus dare: pubes factus pupillus puberi iam factaesorori suaeducenta legati causa sol vit. Q uaesitum est, an tutelaeiudicio consequi possit, quod in alimenta pupillae et mercedes a tutore ex tutela praestitum sit, repondi: existimo, etsi citra magistratuum decretum tutor sororem pupilli sui aluerit et liberelibus artibus (iudicio pupillo aut substitutis pupilli praestare debere.

A nuestro juicio, Juliano deja suficientemente claro en el texto citado que los gastos realizados por el tutor en concepto de alimentos a la hermana, aunque no se hubiesen decretado expresamente por los magistrados, no se le pueden reclamar. La atención de alimentos a la hermana necesitada, como subraya Juliano, no podía dejarse de hacer: existimo, etsi citra magistratuum decretum tutor sororem pupilli sui aluerit et liberelibus artibus (iudicio pupillo aut substitutis pupilli praestare debere. El desarrollo del fragmento en su conjunto puede desviar la atención, como veremos más adelante. De todas formas, cabría pensar fácilmente que al no necesitar la apreciación expresa del magistrado concreto, se desprende implícitamente como una estimación habitual el probable cumplimiento de la atención de alimentos. De hecho, no podrá reclamarse por ninguna vía los gastos efectuados con esta finalidad. El texto parece desvelar otros condicionamientos que podrían llevar a confusión: aparece como punto de partida el hijo, instituido heredero, un legado como dote a su hija, si contraía nupcias dentro de la familia, y la designación de Sempronio como tutor de los mismos. Los cognados de la pupila reclamaron al tutor, ante el magistrado, y éste dispuso: que el tutor diera alimentos a la pupila y los gastos de educación en artes liberales.

La segunda fase del contenido textual comienza cuando se hace púber el pupilo y cumple la obligación impuesta en el legado a favor de la hermana -que también había alcanzado la pubertad-. La cuestión que se le plantea al jurista pretende aclarar la posibilidad que tiene el pupilo de reclamar - por causa de tutela- los alimentos de la pupila y los salarios de los educadores. La respuesta de Juliano parece contundente: Esto no podría suceder de otro modo, no puede dejar de hacerse. Es decir, nada debe pagar el tutor por esta causa de alimentos prestados a la hermana. Las complicaciones exegéticas se multiplican. Veamos algunos ejemplos.

De las alteraciones formales del texto, que no siempre deben suponer una contradicción, haremos referencia a ALBERTARIO ${ }^{4}$, SOLAZZI $^{5}$ y BESELER 6 , entre otros. En este sentido quedaría:

\footnotetext{
${ }^{4}$ ALBERTARIO, E., diritto agli alimenti, cit., pp. 270 y ss.

SOLAZZI, S., Studi sulla tutela, cit., pp.127 y ss

${ }^{6}$ BESELER, G., Beiträgt zur Kritik der römischen Rechtsquellen, cit., 2, p. 42.
} 
D. 27,2,4 (Iulianus, libro XXI digestorum): Qui filium heredem instituerat, filiae dotis nomine, cum in familia nupsisset, ducenta legaverat nec quicquam praeterea, et tutorem eis Sempronium dedit: is a cognatis et a propinquis pupillae perductus (ad magistratum)consulem (cfr.SOLAZZI) iussus est alimenta pupillae et mercedes, ut liberalibus artibus institueretur, pupillae nomine praeceptoribus dare: pubes factus pupillus puberi iam factae sorori suae ducenta legati causa solvit. Quaesitum est, an tutelae iudicio consequi possit, quod in alimenta pupillae et mercedes a tutore (ex tutela) praestitum sit, repondi: existimo, (etsi citra magistratuum- consulum, cfr., SOLAZZI- decretum -si ex consulum decreto-) tutor sororem pupilli sui aluerit et liberelibus artibus instituerit, (cum haec aliter ei contingere non possent), nihil eo nomine tutelae iudicio pupillo (aut substitutis pupilli) praestare- praestari, cfr., BESELER- debere.

ALBERTARIO ${ }^{7}$, considerando en parte las apreciaciones de $\mathrm{SOLAZZI}^{8}$, y sin grandes diferencias con BESELER ${ }^{9}$, realiza una reconstrucción del texto que parece impedir la posibilidad de confirmar la existencia de una obligación de alimentos entre hermanos. Agudamente, nuestro autor destaca lo improbable que debería ser una obligación legal entre el pupilo y la pupila, acogiéndose al tenor literal propuesto por él, es decir, basándose en una interpretación implícita del testamento, en la cual se observaría que el legado previsto estaría subordinado y condicionado a un determinado evento: contraer nupcias dentro de la familia. Si bien, el hecho que señala nuestro autor, como primordial, para llegar a esta conclusión gravita en torno a la interpretación del testamento, y no reflexiona a favor de una supuesta obligación legal precedente; asimismo nuestro estudioso, añade que la ausencia de responsabilidad del tutor es advertida por Justiniano ante el cumplimiento de tales deberes.

A nuestro modo de ver, en ambos casos se trata de una idea latente en las previsiones clásicas como hemos tenido oportunidad de comprobar en un amplio elenco de fragmentos. Por un lado, la exención de responsabilidades respecto al tutor por atender ciertas cargas necesarias, entre las que se encuentran los casos de suministro de alimentos a la madre necesitada o a la hermana, representa, sin duda, un indicio bastante consistente en las propuestas clásicas. Por otra parte, nos parece que el texto reproduce el pensamiento del jurista en sentido lineal, y probablemente, con los agudos recortes señalados por algún sector doctrinal al que ya nos hemos referido, lo único que se acentúa especialmente es la aquiescencia justinianea, pero no se resquebraja a nuestro juicio la esencia clásica del fragmento.

En otro sentido, pero con ciertas analogías al que acabamos de señalar, se muestran las opiniones de otros autores como por ejemplo LAVAGGI ${ }^{10}$, que ni

\footnotetext{
7 ALBERTARIO, E., Sul diritto agli alimenti, cit., pp. 270 y ss.

${ }^{8}$ SOLAZZI, S., Studi sulla tutela, cit., pp.127 y ss.

9 BESELER, G., Beiträgt zur Kritik der römischen Rechtsquellen, cit., 2, p. 42.

${ }^{10}$ LAVAGGI, G., Alimenti (diritto romano), cit., pp. 20 y ss.
} 
siquiera se plantea la necesidad de profundizar en la exégesis textual, al observar que este fragmento se encuentra dentro de los diferentes parágrafos que hablan a favor de la existencia de reciprocidad de una obligación en tema de alimentos entre hermanos (p.e., cita -además del precedente D. 27,2,4-, las siguientes: D. 27,3,1,2 y D. 26,7,13,2). En las confrontaciones doctrinales que avalan nuestra sugerencia encontramos otra de $\mathrm{ZOZ}^{11}$ que, a mi juicio, merece una particular atención. Precisamente se trata de posiciones que toman como punto de partida un adecuado análisis de diferentes textos (D. 27,2,4; D. 27,3,1,2; D. 26,7,13,2; D. 23,3,73,1; D. 24,3,20), algunos ya citados por nosotros, para llegar a una reflexión que puede identificarse con nuestra tendencia. Recuérdese que en D. 27,2,4, se hablaba de un tutor que se encontraba en la necesidad de alimentar a la hermana de su pupilo e instruirla en las artes liberales. Incluso sin que así lo hubieran decretado los magistrados, no tendría que responder de estos gastos en un supuesto juicio de tutela frente al pupilo o los sustitutos del pupilo. En esos términos se expresaba Juliano. En la gestión del patrimonio pupilar, estas actuaciones del tutor gozan de una complacencia jurídica sobradamente reconocida: estas atenciones no podían dejarse de hacer o cumplir. Más bien, se puede ejercitar contra el tutor la acción de tutela, si se hubiera desatendido este deber: D. 27,3,1,2: ...posse cum tutore agi tutelae, si tale afficium praetermiserit.

Una asumible matización de $\mathrm{LONGO}^{12}$ afirma que se habla claramente de una obligación de alimentos en este último fragmento mencionado (en relación a la hermana y la madre):

D . 27,3,1,2 (U Ipianus Libro XXX VI ad edictum) Sed et si non mortis causa donaverit tutore auctore, idem I ulianus scripsit pleros que quidem putare non valere donationem, et plerumque ita est: sed nonnullos casus posse existere, quibus sine reprehensione tutor auctor ft pupillo ad deminuendum, decreto scilicet interveniente: veluti si matri aut sorori, quae aliter se tueri non possunt, tutor alimenta praestiterit: nam cum bonae fidei iudicium sit, nemo feret, inquit, aut pupillum aut substitutum eius querentes, quod tam coniunctae personae al itae sint: quin immo per contrarium putat posse cum tutore agi tutelae, si tale officium praetermiserit.

\section{DEBER EXPLÍCITO: PRESTAR LOSALIMENTOS NECESARIOS ALAHERMANA (O HERMANO)}

Es un deber, ayudar a la hermana y a la madre del pupilo con la correspondiente prestación de los alimentos necesarios, y como se desprende de las afirmaciones de Juliano, nadie podrá quejarse, reclamar, o tolerar que se incumpla este tipo de atención a personas tan allegadas. Evidentemente, como expresa Juliano, se pueden dar algunos

\footnotetext{
${ }^{11}$ ZOZ, M.G., In tema di obbligazioni alimentari, cit., p. 342.

${ }^{12}$ LONGO, G., Sul diritto agli alimenti, cit., p.343.
} 
casos en los que el tutor tenga que dar su autoridad sin que se le pueda reprochar su actuación. En este supuesto nos encontramos cuando se refiere al tutor que proporciona alimentos a la hermana y a la madre. Es más, el descuido en el cumplimiento de este deber del tutor - alimentar a la madre y a la hermana del pupilo- le puede suponer una demanda por la acción de tutela. Hemos prescindido del análisis de las donaciones inválidas, aludidas en el fragmento, para centrarnos en los casos en los que se ve obligado el tutor a prestar su autoridad: dar alimentos a la hermana y la madre.

El texto, al menos en la sustancia, afirma acertadamente $\mathrm{LONGO}^{13}$ parece clásico. Una revisión exegética de las fuentes no debilita su opinión acerca de la raíz clásica de la obligación de alimentos entre hermanos. Incluyendo los fragmentos que podrían emanar indicios de confusión debido a los enunciados justinianeos:

D. 26,7,13,2 (Gaius libro XXII ad edictum) In solvendis legagtis et fidei commissis attendere debet tutor, ne cui non debitum solvat, nec nuptiale munus matri pupilli vel sorori mittere. Aliud est, si matri forte aut sorori pupilli tutor ea quae ad victum necessaria sunt praestiterit, cum semet ipsa sustinere non possit: nam ratum id habendum est: nec enim eadem causa est eius, quod in eam rem ipenditur et quod muneris legatorummve nomine erogatur.

D. 26,7,12,3 (Paulus libro XXXVIII ad edectum) C um tutor non rebus dumtaxat, sed etiam moribus pupilli praeponatur, imprimis mercedes praeceptoribus, non quas minimas poterit, sed pro facultate patrimonii, pro dignitate natalium constituet, al imenta servis libertisque, nonnumquam etiam exteris, si hoc pupillo expediet, praestabit, soll emnia munera parentibus comgnatisque mittet sed non dabit dotem sorori alio patre natae, etiamsi al iter ea nubere non potuit nam etai honeste, ex liberalitate tamen ft, quae servanda arbitrio pupilli est.

Por su parte $\mathrm{LAVAGGI}^{14}$, sin entrar en fundamento, manifiesta la misma proclividad a creer en la prestación de alimentos entre hermanos con factura clásica, constituyendo lo expresado en el ya citado D. 27,3,1,2 $2^{15}$, otro de los textos que ha utilizado nuestro autor para confirmar su reflexión.

Entre los autores que en mi opinión interesa destacar también, por demostrar, o al menos afirmar con solidez lo infructuoso que puede ser sospechar de los referidos textos, D. 27,2,4 $4^{16}$ y D. 27,3,1,2, continuaremos con las opiniones de $\mathrm{ZOZ}^{17}$. Se trata de dos textos, "insospettabili", afirma nuestra autora. No existen, por tanto, consideraciones válidas que puedan demostrar que tanto las previsiones de Juliano como el pensamiento de Ulpiano sean erróneos, más bien, todo lo contrario; es decir se reproduce aquí para nuestra romanista el pensamiento exacto e inequívoco del jurista.

\section{LARELACIÓN DE ALI MENTOS PODRÍA CONSI DERARSE}

\footnotetext{
${ }^{13}$ LONGO, G., Sul diritto agli alimenti, loc. cit.

${ }^{14}$ LAVAGGI, G., Alimenti (diritto romano), cit., pp. 20.

${ }^{15}$ D. 27,3,1,2 (Ulpianus Libro XXXVI ad edictum).

${ }^{16}$ D. 27,2,4 (Iulianus, libro XXI digestorum).

${ }^{17}$ ZOZ, M.G., In tema di obbligazioni alimentari, cit., p. 342.
} 


\section{COMO UNAAUTÉNTICARELACIÓN JURÍDICA}

Una síntesis de los datos más significativos y elocuentes de los referidos textos ya la hemos realizado, si bien, nos gustaría añadir con nuestra estudiosa, algunas de las enseñanzas complementarias que pueden extraerse. Partiendo de lo concerniente al decreto de condena de alimentos, observa $\mathrm{ZOZ}^{18}$ acertadamente: la importancia de la sanción por el derecho extraordinario de la obligación de alimentos entre hermanos -ya desde Juliano, uno de los más eminentes impulsores de la ciencia jurídica-. Asimismo, que la relación de alimentos se considera como una verdadera y propia relación jurídica. El eventual incumplimiento del pupilo, aunque sea por medio del tutor, lo hace responsable en las confrontaciones del pupilo mismo. Se da por admitido que respecto a la madre, subsiste la obligación de alimentos (asumiendo, como hemos visto, la equiparación de la obligación que en el texto de Ulpiano se presenta, es decir, madre o hermana. Y, finalmente respecto a la hermana, en consonancia con lo que hemos visto precedentemente. Todas las puntualizaciones que hemos acumulado encuentran fiel acogida y confirmación también en D. 26,7,13,2 ${ }^{19}$ (LAVAGGI $^{20}$, LONGO ${ }^{21}, \mathrm{ZOZ}^{22}$, entre otros).

La sugerencia de $\mathrm{LONGO}^{23}$ nos parece muy significativa, sobre todo teniendo en cuenta que al principio de su comentario a este respecto, incluía el siguiente texto que analizaremos entre los de dudosa interpretación, y quizá, con enunciados propiamente justinianeos. Consecuencias que, con matices, no impiden a nuestro autor reafirmar el carácter de obligación de la prestación de alimentos entre hermanos dentro de la perspectiva clásica.

Así pues, escribe nuestro autor ${ }^{24}$, la obligación de alimentos entre hermanos y hermanas aparece afirmada, si hoc pupillo expediat ${ }^{25}$. Restricción que sobre todo podría constituir una prueba contra la existencia de un principio jurídico, propio del nuevo derecho, que afirmara la pretendida obligación de alimentos entre hermanos. Asimismo, conviene recordar con nuestro autor que en este tipo de actuaciones, tanto en las concepciones clásicas como en los enunciados justinianeos, principalmente se habla de daciones, suministros, repartos voluntarios o socialmente convenientes, más que de una obligación podría confirmar la existencia de dos perspectivas: la primera que siempre estará presente a la hora de las aportaciones de sustento es el

\footnotetext{
${ }^{18}$ ZOZ, M.G., In tema di obbligazioni alimentari, cit., p. 342.

${ }^{19}$ D. 26,7,13,2 (Gaius libro XXII ad edictum).

${ }^{20}$ LAVAGGI, G., Alimenti (diritto romano), , cit., pp. 20.

${ }^{21}$ LONGO, G., Sul diritto agli alimenti, cit., p.343.

${ }_{22}$ ZOZ, M.G., In tema di obbligazioni alimentari, cit., p. 343.

${ }^{23}$ LONGO, G., Sul diritto agli alimenti, loc. cit.

${ }^{24}$ LONGO, G., Sul diritto agli alimenti, loc. cit.

${ }^{25}$ Cfr. D. 26,7,12,3
} 
nivel de facultades patrimoniales para su cumplimiento, pro facultate patrimonii; la segunda, que recalcar el carácter de la voluntariedad, como indicio de la inexistencia de obligación clásica, no parece representar un argumento válido, sobre todo teniendo en cuenta la analogía de los enunciados justinianeos a este respecto. Cabría recordar ahora, la claridad con la que se expresa $\mathrm{LONGO}^{26}$ cuando habla de la obligación de alimentos, en relación a la hermana, en su comentario y defensa de la sustancia clásica del fragmento recogido en D. 27.3.1.2.

De este modo, queremos resaltar con SACHERS ${ }^{27}$, del conjunto de textos analizados por él (en el que se conjugan matices clásicos y enunciados justinianeos), las expresiones extraídas de los mismos, como imposibilidad de mantenerse por uno mismo, pobreza o necesidad, incapacidad, como consecuencia de impedimentos físicos o de otra naturaleza, como condición para la posible pretensión -en suma, previa comprobación de los presupuestos necesarios para hacer valer este derecho-. Estas alusiones no dejan de confirmar, a nuestro entender, la conservación del espíritu legislativo impulsor de las atenciones clásicas respecto a la prestación, y, especialmente en este epígrafe podríamos dirigirlas al carácter de la obligación recíproca entre hermanos.

En consecuencia y sin tener que recurrir ahora a una lectura global de los textos en los que se puede dispersar en ocasiones el discurso de nuestros juristas, seguiremos analizando una de las disposiciones ya referidas, en la que desde nuestro punto de vista también se vislumbran adecuadamente los datos más sobresalientes que nos permitirán completar y configurar mejor el pensamiento de Gayo (D. 26,7,13,2 2) ${ }^{28}$. Con ciertos matices que podremos compartir con $\mathrm{ZOZ}^{29}$, retomamos nuestro análisis del fragmento de Gayo: si el tutor hubiera suministrado a la madre o a la hermana del pupilo, lo que es imprescindible y necesario para el sustento y alimentación, -es decir, quae ad victum necessaria sunt-, en los supuestos en los que no pudieran mantenerse por sí mismas - praestiterit quum semet ipsa sustinere non possit-, quedará liberado de responsabilidades añadidas por haber ejecutado estas atenciones (obligaciones) dentro del marco de la validez jurídica. La diligencia del tutor -en el cumplimiento que por imperativo legal se establece en el fragmento respecto a los legados y fideicomisos-, debe estar presente con la finalidad de evitar que los pagos se hagan a personas que no tengan tal derecho, o bien, puedan encuadrarse en el campo de los regalos nupciales a la madre o la hermana del pupilo; lo que sí implicaría por consiguiente la invalidez de las actuaciones del tutor y las probables responsabilidades del mismo. El cuidado y la

${ }^{26}$ LONGO, G., Sul diritto agli alimenti, loc. cit.

${ }^{27}$ SACHERS, E., Das Recht auf Unterhalt in der römischen Familie der klass. Zeit, p. 330 n. 4.

${ }^{28}$ D. 26,7,13,2 (Gaius libro XXII ed.)

${ }^{29}$ ZOZ, M.G., In tema di obbligazioni alimentari, cit., pp. 343 y ss. 
atención especial del tutor viene provocada por la gran diferencia que se advierte por el jurista en cuanto a las disposiciones patrimoniales y los gastos que representan -no está en el mismo caso lo que se gasta así (en concepto de alimentos necesarios), y lo que se gasta en concepto de regalos y fideicomisos-. Este matiz, supone una explicación bastante esclarecedora y perfectamente puede contribuir implícitamente, a nuestro juicio, a evitar el confusionismo de los gastos, teniendo en cuenta que pueden tener una misma finalidad (piénsese en el legado de alimentos al que ya nos referimos en epígrafes anteriores). En otras palabras, aquí puede observarse, con carácter independiente, que se trata de dar validez a los gastos ocasionados específicamente para el sustento de la hermana (y la madre), asumidas como una conveniencia con carácter de obligación que no supondrá responsabilidades añadidas para el tutor, sino más bien todo lo contrario. Como es sabido, la conveniencia de este tipo de actuaciones en concepto de alimentos encuentra cobertura legal en numerosas explicaciones particulares aportadas por diferentes vías jurídicas. No exigir responsabilidad jurídica al promotor de las actuaciones (tutor), no sólo implicaría conveniencia, sino más bien exigencia jurídica. En estos términos nos hemos pronunciado en líneas anteriores cuando destacábamos, en atención a lo dispuesto en D. 27,3,1,2, que se podría demandar al tutor por el incumplimiento de este deber: dar alimentos a la hermana o a la madre del pupilo que no puedan valerse por sí mismos.

D. 26,7,13,2 (G aius, libro XXII ad edictum) In solvendis legatis et fideicommissis attendere debet tutor, ne cui non debitum solvat, nec nuptiale munus matri pupilli vel sorori mittere. Aliud est, si matri forte aut sorori pupilli tutor ea quae ad victum necessaria sunt praestiterit, cum semet ipsa sustinere non possit: nam ratum id habendum est: nec enim eadem causa est eius, quod in eam rem ipenditur et quod muneris legatorummve nomine erogatur.

D. 27,3,1,2 (U Ipianus Libro XXXVI ad edictum) Sed et si non mortis causa donaverit tutore auctore, idem I ulianus scripsit pleros que quidem putare non valere donationem, et plerumque ita est: sed nonnullos casus posse existere, quibus sine reprehensione tutor auctor ft pupillo ad deminuendum, decreto scilicet interveniente: veluti si matri aut sorori, quae aliter se tueri non possunt, tutor alimenta praestiterit: nam cum bonae fidei iudicium sit, nemo feret, inquit, aut pupillum aut substitutum eius querentes, quod tam coniunctae personae al itae sint: quin immo per contrarium putat posse cum tutore agi tutelae, si tale officium praetermiserit.

En definitiva, advertimos aquí unos modelos de afirmación clara que se amoldan a nuestras apreciaciones, y que, como subraya $\mathrm{ZOZ}^{30}$, acerca del primero de los textos (D. 26,7,13,2), "rientra invece nei poteri gestori l'adempimento alla prestazione alimentari, quando madre e sorella siano in stato di bisogno"; además, prosigue nuestra autora, "anzituto l'accento sulle condizioni disagiate fa pensar al vero e proprio obbligo alimentare fundato sul rapporto familiare, que trova appunto causa esclusivamente da tali condizioni".

${ }^{30}$ ZOZ, M.G., In tema di obbligazioni alimentari, loc. cit. 
La mujer, para atender las necesidades de alimentos de los hermanos ${ }^{31}$ que se encontraban en estado de necesidad, puesto que se trata de una causa justa y honesta, como nos dice Paulo en D. 24,3,20, podía cobrar la dote constante matrimonio:

D. 24,3,20 (Paulus, libro V II ad Sabinum): Q uamvis mulier non in hoc accipiat constante matrimonio dotem, ut aes alienum solvat, aut praedia idonea emat, sed ut liberis ex alio viro egentibus, aut fratribus, aut parentibus consul eret, vel ut eos ex hostibus redimeret, quia iusta et honesta causa est, non videtur male accipere; et ideo recte ei solvitur, idque et in filia familias observatur.

\section{REFLEXIONESY OBSERVACIONES}

Este aspecto podría implicar una previsión jurídica seria, reservada a la mujer que se encontraba en esta situación, para cumplir su parte de obligación en tema de alimentos también respecto a los hermanos. Las divergencias exegéticas han saturado indiscutiblemente los resultados doctrinales, sobre todo cuando se ha mirado el texto citado desde la perspectiva del paralelismo pauliano afirmado en otro de sus fragmentos (D. 23,3,73,1), al que también se le atribuyen algunas enmiendas justinianeas. Agudamente nos recuerda $\mathrm{ZOZ}^{32}$, en relación a los dos textos mencionados del mismo autor -que además se sostienen recíprocamente aunque hayan sido tratados en obras no coincidentes-, resulta inverosímil pensar en una intervención sustancial por parte de los compiladores. Se trata de fragmentos que no son tratados en la misma masa (Papinianea, D. 23,3,73,1; Sabinianea, D. 24,3,20); se encuentran en títulos y libros distintos; en una sede que no es la propia de los alimentos-. En consecuencia, parecería inverosímil, que con la finalidad de introducir una nueva regla en tema de alimentos, las dos subcomisiones diferentes, y en sede distinta a la propia de alimentos, se hayan dedicado a interpolar, y justamente en el mismo sentido, dos textos completamente diversos que provienen del mismo autor. Una explicación suya muy asumible puede simplificar la cuestión: Los textos refieren los mismos principios porque son los conceptos del mismo autor.

\footnotetext{
${ }^{31} \mathrm{~L}$ O de los hijos de otro marido, o de los ascendientes.

${ }^{32}$ ZOZ, M.G., In tema di obbligazioni alimentari, cit., pp. 344.
} 
D. 23,3,73,133 (Paulus, libro II sententiarum): M utus surdus caecus dotis nomine obligantur, quia et nuptias contrahere possunt. $M$ anente matrimonio non perditurae uxori ob has causas dos reddi potest: ut sese suosque alat, ut fundum idoneum emat, ut in exilium vel in insulam relegato parenti praestet alimonia, aut ut egentem (virum ${ }^{34}$ ) fratrem sororemve sustineat.

A las ya mencionadas observaciones podrían añadirse otras muchas que contribuirían a disipar las inseguridades y ambigüedades latentes; si bien, a nuestro propósito, a pesar de la desconexión de los textos referidos de su propio contexto específico, lo que podría provocar una especie de desviación del sentido de los mismos, podríamos afirmar el aspecto de la reciprocidad entre hermanos - si bien, en determinados casos, al menos implícita-; el carácter de obligación de la prestación precedida de un conjunto armónico de estimaciones jurídicas afirmativas; que los receptáculos de duda que puedan desprenderse de los dos últimos textos transcritos (de diferentes libros del Digesto, pero del mismo autor), independientemente de la forma expositiva, reproducen adecuadamente, y sin carácter de excepción o aplicación limitada, la estructura lineal del pensamiento del discípulo de Quinto Cervidio Escévola, Paulo. Un jurista perfectamente adherido a la realidad de la práctica cotidiana, adaptado y proclive por cauce natural al continuo esfuerzo imperial por dotar de una fisonomía más completa al instituto de la prestación de alimentos.

\section{ENFOQUE ROMANÍSTICO SOBRE A SOLIDARIEDADE NATURAL E JURÍDICA DAPRESTAÇÃO DE ALIMENTOS ENTRE IRMÃOS}

Resumo: Neste artigo destacamos o caráter obrigacional da prestação de alimentos entre irmãos, provavelmente já existente desde a época clássica mais avançada. Nossa proposta encontra apoio num conjunto de entendimentos jurisprudenciais e na análise profunda das principais fontes jurídicas.

Palavras-c have: Alimenta. V ictus. F ilius.

${ }^{33}$ Cabría recordar que SACHERS, E., Das Recht auf Unterhalt in der römischen. Familie der klass. Zeit, cit., pp. 341 n. 2 y 3 , admitía la clasicidad del texto.

${ }^{34}$ ZOZ, M.G., In tema di obbligazioni alimentari, cit., pp. 344, nos recuerda además la propuesta por MOMMSEN, T., ad. 1., <<filium ex alio viro (?) >> fratrem sororemve sustineat, adecuadamente rebatida por ella. 